\title{
UN PUNTO DE VISTA MÉDICO SOCIAL SOBRE LA DEPENDENCIA DE LAS DROGAS *
}

\author{
Francisco Javier Yuste Grijalba \\ (Jefatura Provincial de Sanidad de Pontevedra)
}

Los distintos conceptos que han sido utilizados en el campo de la dependencia de las drogas han cristalizado en la actual concepción sancionada por la Organización Mundial de la Salud (OMS) que resalta su unidad teórica y práctica y sus diferentes tipos sindrómicos. La epidemiología de las dependencias se presenta articulada más en datos cualitativos que en su exacta cuantificación, dada la dispersión e inactualidad de ellos. Las teorías más significativas que operan en este campo se presentan aquí, particularmente las que se han enunciado desde las perspectivas sociológicas. El análisis de la anomia de Merton es particularmente claro en su aplicación al caso. Finalmente, este estudio presenta unas consideraciones preventivas y terapéuticas sobre las dependencias, insistiendo en la necesidad de su consideración como enfermedades $\mathrm{y}$, consecuentemente, en el tratamiento médico como base de la recuperación de los dependientes.

* Debo agradecer a J. M. de Miguel sus certeras observaciones al borrador de este trabajo, asimismo a $\mathrm{C}$. Baquero su esfuerzo mecanográfico. 

La línea de salida del problema de las dependencias se sitúa en la existencia de numerosas sustancias químicas cuyos efectos sobre el sistenia nervioso del ser humano son buscados por éste hasta ligarse a aquéllas de modo indisoluble. El fenómeno es muy antiguo, el peyote y otros alucinógenos entre los indios precolombinos, la raíz de la Iboga en el África negra, la amanita entre los Vedas, son ejemplo de ello. Hoy el problema es alarmante; en algunos países la preocupación más considerable es el alcohol, en otros los sedantes y estimulantes.

La mera existencia de sustancias psicotropas no constituye en sí mismo un problema, ni siquiera la ligazón anancástica entre sustancia y usuario. El problema surge por las consecuencias patológicas a que conduce el uso continuado de aquéllas. En efecto: daño crónico en el sistema nervioso, intoxicaciones mortales por sobredosis, septicemias, etc., en el terreno individual; delitos en la búsqueda «al-precio-que-sea» de la sustancia y desintegración familiar, accidentes de tráfico en la esfera comunitaria y consecuencias directas de la relación hombre-droga hacen que las dependencias sean un problema social en el mundo actual.'

\section{Conceptos y terminología}

Si tenemos en cuenta la variedad de productos que son capaces de ligar a la persona a su consumo, las diferencias individuales en las respuestas, que lo que se trata de conceptualizar es la interrelación de hombre y droga, podremos comprender que haya sido difícil situar el marco teórico de las dependencias. En definitiva, lo que todo concepto intenta es expresar sintéticamente una realidad; la evolución en los conceptos traduce la profundización en su análisis, cuyo recorrido nos puede ayudar para mejor conocer aquélla.

1. S. A. Clausen, «Drug addiction», pp. 193-235, en R. K. Merton y R. A. Nisbet (eds.), Contemporary Social Problems (Nueva York: Harcout, 1966), $2 .^{2}$ ed. 
El punto de partida de la evolución conceptual se sitúa en el $V$ Informe del Comité de Expertos en Drogas Toxicomaningenas ${ }^{2}$ en el que se definió con carácter general, aunque provisionalmente, el fenómeno de la toxicomanía. La aplicación al estudio del alcoholismo puso de manifiesto la inoperancia de esta primera aproximación. A la luz de los datos entonces disponibles, se consideró que el alcohol debía ser clasificado en un lugar intermedio entre las drogas que causan toxicomanía y las que simplemente causan hábito. ${ }^{3}$ Todo ello colaboró a separar artificiosamente toxicomania de babituación. Toxicomanía sería un estado de intoxicación transitoria o crónica producida por el consumo repetido de una droga natural o sintética. Sus características incluyen: un deseo o necesidad irresistible (compulsión) para continuar tomando la droga y para obtenerla de cualquier modo; tendencia a incrementar la dosis: es decir, dependencia psíquica (psicológica) y generalmente física de los efectos de la droga; y efectos deletéreos para la sociedad y el individuo. Mientras que babituación consistiría en una condición resultante del repetido consumo de una droga. Sus características serían: deseo (pero no compulsión) a continuar tomando la droga para procurarse las sensaciones de bienestar que produce; poca o ninguna tendencia a aumentar la dosis; algún grado de dependencia psíquica, pero ausencia de dependencia física y por tanto de síndrome de abstinencia; efectos deletéreos, si alguno, en el individuo. ${ }^{4} \mathrm{La}$ clave para la distinción estaría en la dependencia física y su secuela el síndrome de abstinencia ligados a la toxicomanía, y la dependencia psíquica ligada al hábito.

No sólo lo confuso de las diferencias sino también la continuada aparición de nuevos agentes y el uso impropio, como sinónimos, de aquellos dos términos impulsó a su sustitución por uno único: dependencia de las drogas, ${ }^{5}$ que vendría definido genéricamente por el estado originado por la administración o consumo repetidos de una sustancia, y específicamente por el tipo de sustancia hacia el que se ha establecido la dependencia. El planteamiento tendría en buena lógica que ser unitario

2. Organización Mundial de la Salud, Serie de Informes Técnicos, 57 (Ginebra OMS, 1952).

3. OMS, Serie de Informes Técnicos, 87 (Ginebra: OMS, 1954), loc. cit. (Ginebra: OMS, 1955). Como en tantas ocasiones debajo había la valoración ética de que ser morfinómano es peor que ser alcohólico. Como ha señalado Jellinek, «para evitar cualquier mácula del alcoholismo, que está relacionado con una costumbre social altamente considerada, con nada que sugiera el menospreciado uso de los narcóticos». The Disease Concept of Alcobolism (New Haven: Hillhouse, 1960).

4. OMS, Serie de Informes Técnicos, 116 (Ginebra: OMS, 1957).

5. OMS, Serie de Informes Técnicos, 274 (Ginebra: OMS, 1964). 
y así fue realizado por Eddy et alii, ${ }^{5}$ y sancionado por el Grupo Científico de la OMS sobre Evaluación de las drogas que causan dependencia. El término dependencia expresa una especial relación hombre-droga, por la que existe en aquél una tendencia anancástica a utilizar ésta. ${ }^{8}$ Ahora bien, esta relación es de tal amplitud que necesita ser calificada con mayores matices. Así, del género dependencia pueden desgajarse las especies dependencia psíquica y física. La primera se constituye por un impulso psíquico que lleva a tomar, periódica o continuamente, el fármaco para experimentar un placer o para evitar un malestar. 'La segunda es el estado de adaptación que se manifiesta por la aparición de intensos trastornos físicos cuando se suspende la toma del fármaco; estos trastornos, llamados síndrome de abstinencia, son de naturaleza física o psíquica y característicos de cada tipo de fármaco. ${ }^{10}$

La dependencia puede ir o no acompañada de tolerancia, fenómeno acompañante de muchos medicamentos caracterizado por la atenuación de las respuestas a la misma cantidad de sustancia, lo que lleva al aumento de la dosis para conseguir el mismo efecto." Aun cuando dependencia y tolerancia no van necesariamente unidas, cuando aparece ésta se induce aquélla con mayor facilidad.

Los tres elementos fenomenológicos que se pueden encontrar en el círculo vicioso de la dependencia dan lugar mediante su combinación en mayor o menor grado a cuadros clínicos diferentes que corresponden etiológicamente a drogas diferentes. La observación de este hecho ha permitido especificar la dependencia de acuerdo con la droga que la determina y delimitar, de la enorme cantidad de sustancias capaces de generar dependencias, ${ }^{12}$ unos cuantos tipos afines:

6. N. B. Eddy, H. Halbach, H. Jibell y M. Seevens, «Drug Dependence: Its Significance and Characteristics», Bulletin Organisation Mondiale de la Santé, 32 (1965), pp. 721-755.

7. OMS, Serie de Informes Técnicos, 287 (Ginebra: OMS, 1964).

8. OMS, Serie de Informes Técnicos, 363 (Ginebra: OMS, 1967).

9. N. B. Eddy et al., op. cit., p. 273. También puede verse la OMS, Serie de Intormes Técnicos, 407 (Ginebra: OMS, 1969). Para juzgar en la práctica la presencia de dependencia psíquica es importante determinar hasta qué punto el uso de las drogas parece ser un factor importante de la organización de la vida y adquirir prioridad sobre el uso de otros mecanismos de defensa competitivos. El uso de una droga es un factor importante en la vida si se dedica demasiado tiempo a su ob. tención, uso o discusión sobre ella. El consumo es prioritario si el individuo responde a las situaciones difíciles de la vida con la ingestión de la droga.

10. N. B. Eddy et al., op. cit., p. 724.

11. Idem.

12. En sentido amplio el café, el té, el tabaco, pueden considerarse sustancias 
(1) Alcobol-barbitúricos: étanol, barbitúricos y otros fármacos con efectos sedantes como el hidrato de cloral, el clordiacepóxido, el diazepam, el meprobramato y el metacuelona.

(2) Anfetamina: anfetamina, dexanfetamina, metanfetamina, metilfenidato y fenmetracina.

(3) Cannabis: preparaciones de cannabis sativa L como marihuana (bang, dagga, kif, maconha), ganka y hashish (charas).

(4) Cocaina: Cocaína y hojas de coca.

(5) Alucinógeno: lisérgida (LSD), mescalina y silocibina.

(6) Khat: preparaciones de Catha edulis Forssk.

(7) Opiáceos (morfina): opiáceos como la morfina, la heroína, la codeína y productos sintéticos; efectos morfínicos como la metadona y la petidina.

(8) Solvente volátil (inhalable): tolueno, acetona y tetracloruro de carbono. $^{13}$

Cada uno de los males tiene un patrón distinto de acuerdo con una mayor o menor participación de dependencia física, psíquica y tolerancia.

\section{Epidemiología de las dependencias}

Las dependencias, como cualquier problema que afecte a la salud pública, pueden ser estudiadas con la metodología epidemiológica para, observando las variaciones en la distribución de las mismas en la población e induciendo las causas de la misma, tratar de eliminarlas en cuanto problema de salud pública.

La epidemiología de la farmacodependencia puede compararse a la de una enfermedad transmisible, pues en ambas existen huéspedes actuales y potenciales con diversos grados de resistencia al trastorno, y los factores ambientales colaborarán a que la exposición al riesgo sea efectiva; está presente un «agente» de infección - bacilo de Koch en la tuberculosis, heroína o alcohol en estas dependencias- o existe un vector de la enfermedad: el instigador en la farmacodependencia. Como en el caso de las

que generan dependencia. No cabe duda del daño a la salud producido por el tabaco, incluso podría hablarse de una dependencia psíquica en los grandes fumadores, pero mientras debamos exigir una influencia en el sistema nervioso central para hablar de drogas, la nicotina no lo será. Véase OMS, Serie de Informes Técnicos, 516, 526 (Ginebra: OMS, 1973).

13. N. B. Eddy et al., op. cit., p. 725. También OMS, Serie de Informes Técnicos, 287 (Ginebra: OMS, 1964); loc cit., 526 (Ginebra: OMS, 1973). 
enfermedades venéreas hay una «conducta de búsqueda» por parte del huésped. ${ }^{14}$

La información epidemiológica sobre la dependencia es incompleta y dispersa; así y todo podemos agrupar en los siguientes epígrafes los datos de los que extraer conclusiones operativas: Cuantificación del problema y tendencia secular, Variables de lugar, Características de las personas dependientes.

Las fuentes de los datos a valorar en estos epígrafes proceden de los registros nacionales e internacionales de producción y consumo, los registros hospitalarios sobre ingresos por causa de enfermedades psíquicas o físicas vinculadas a la dependencia y de encuestas sobre consumo realizadas en la población. Como es claro, el valor de estos datos es limitado, pero puede facilitarnos referencias de extensión y tendencia.

Utilizando la fórmula de Jellinek ${ }^{15}$ que relaciona las cirrosis hepáticas con el alcohol, González Aguilera ${ }^{16}$ cifró en $2,5 \%$ el número de alcohólicos de la población española, cifra consistente con el 3,6 señalada por Viñes en la población de Navarra ${ }^{17}$ y con las cifras de cirrosis encontradas por J. García en las historias hospitalarias de cinco grandes hospitales españoles. ${ }^{18} \mathrm{La}$ frecuencia del alcoholismo por 100 personas mayores de 20 años en los países y años que se citan es, de acuerdo con la fórmula de Jellinek, la siguiente:" Suiza (1953) 2,1; Inglaterra (1960) 1,1; Dinamarca (1948) 1,9; Francia (1951) 5,2; Chile (1950) 3,6; Finlandia (1955) $1,1$.

En las conclusiones de un Estudio Epidemiológico sobre el alcobolismo en España realizado por J. Santo Domingo, C. Valenciano, y F. Alonso ${ }^{20}$ sobre una muestra de 4.201 personas de Madrid, Murcia y La Coruña leemos: «Existe un 18-24\% de la población que consume más de $100 \mathrm{ml}$ de alcohol absoluto diario, que sanitariamente es exce-

14. D. C. Cameron, «La lucha contra el abuso del alcoholismo y las drogas», Crónica de la Organización Mundial de la Salud, 23 (1969): 171-180.

15. E. M. Jellinek, op. cit. Esta fórmula se expresa por $A=P . D / K$ donde $A$ es el número de alcoholismo, $D$ las defunciones por cirrosis y $K$ una constante cifrada en 0,694 .

16. A. G. Aguilera, «El alcoholismo en España», VII Congreso Espñol de Neuropsiquiatría (Pamplona: mimeo, 1962).

17. J. Viñes, Alcoholismo (Pamplona, Navarra: Publicaciones del Instituto de Sanidad, 1958).

18. J. García, «La cirrosis alcohólica en España», pp. 109-124, en PANAP (ed.), Alcobolismo y otras toxicomanias (Madrid: PANAP, 1970).

19. OMS, Serie de Informes Técnicos, 363 (Ginebra: OMS, 1967).

20. J. Santo Domingo, C. Valenciano y F. Alonso, Estudio epidemiológico sobre el alcobolismo en España (Madrid: PANAP, 1966). 
sivo. Deben ser considerados alcohólicos el $8,7 \%$ de la muestra total; 4,4 en Murcia; 7,9 en Madrid; $14 \%$ en La Coruña.» El Gabinete de estudios de la Dirección General de Sanidad cifra el número de alcohólicos hacia 1963 entre un mínimo de 823.543 casos, aplicando la fórmula de Jellinek, y un máximo de 2.141.000 utilizando los porcentajes de Santo Domingo. ${ }^{21}$

\section{TABla 1}

Consumo medio diario de alcohol absoluto mayor de $150 \mathrm{ml}$. (tasas por 100.000 habitantes de más de 25 años, 1970)

\begin{tabular}{|c|c|c|}
\hline Francia & 9.050 & Rep. Dem. Alemana ........ 2.760 \\
\hline Italia & 7.390 & USA $\ldots . . . . .$. \\
\hline España & 5.350 & Dinamarca \\
\hline Rep. Fed. Alemana & 4.820 & Gran Bretaña \\
\hline Portugal $\quad \ldots \ldots \ldots \ldots \ldots \ldots \ldots$ & 4.690 & $\ldots 1.960$ \\
\hline URSS $\ldots \ldots \ldots \ldots \ldots \ldots \ldots \ldots$ & 4.290 & ... 1.870 \\
\hline Austria & 3.690 & …......... 1.830 \\
\hline Bélgica & 3.650 & Noruega . \\
\hline
\end{tabular}

Fuente: I. De Lint. «Epidemiology of alcoholismo» I Conferencia Internacional sobre Alcobolismo (Londres, 1973).

En la Tabla 1, utilizando los datos de Lint, ${ }^{22}$ se pueden comparar los resultados obtenidos sobre consumo de alcohol absoluto en diversos países. Lo cual, puesto en relación con las tablas de Landerman ${ }^{23}$ que relacionan consumo medio con número estimado de alcohólicos, situaría la cifra española entre $2 \%$ y $4 \%$ de la población.

Los trabajos publicados sobre ingresos en hospitales psiquiátricos de personas afectas por psicosis alcohólica -delirium tremens u otras-, demencia alcohólica o intoxicación aguda mencionan el aumento de los ingresos en línea ascendente. ${ }^{24}$ $39-40$.

21. G. Clavero, «Datos estadísticos sobre alcoholismo», Salud Mental, 4 (1969):

22. J. de Lint, «Epidemiology of Alcoholism», Actas de la Conferencia Internacional sobre Alcobolismo (Londres: 1973).

23. S. Landerman, Alcool, alcoolisme, alcoholisation (París: PUF, 1964).

24. Así, Villacian en Valladolid de $6,1 \%$ de alcohólicos respecto al total de 1930 pasa al $12,9 \%$ en 1950 , y a $20 \%$ en 1967; Pelaz en Madrid de un $1 \%$ en 1946 a 16,4\% en 1955; Santo Domingo en el hospital de agudos de $4 \%$ en 1944 a $18 \%$ en 1960; y Murcia Valcarcel en Barcelona de 5,4\% en 1939 a $10,6 \%$ 
Con respecto a las características de las personas alcohólicas, y atendiendo a los datos publicados por Santo Domingo, ${ }^{25}$ podemos decir que el alcoholismo es fundamentalmente un fenómeno masculino, entre 30 y 40 años de edad. La mujer ha comenzado a no ser excepción a esta ley: los datos que sobre toxicomanías en España obran en nuestro poder son poco precisos. Los más exactos se refieren a los enfermos fichados por usar estupefacientes para alguna dolencia alógena y necesitar en consecuencia carnet de extradosis para conseguir su medicación. Los toxicómanos registrados por enfermedad en España son: ${ }^{26}$

\begin{tabular}{llll}
\hline & 1968 & 1970 & 1972 \\
\hline Varones & 337 & 281 & 257 \\
Mujeres & 563 & 474 & 409 \\
\hline
\end{tabular}

No tienen, a nuestro juicio, significación los datos de toxicómanos por hábito que transcribe el Anuario Estadistico Español. Éstos son (números absolutos):

\begin{tabular}{crrr}
\hline & 1968 & 1970 & 1972 \\
\hline Varones & 49 & 34 & 41 \\
Mujeres & 113 & 97 & 118 \\
\hline
\end{tabular}

En la Tabla 2 se ofrecen las informaciones suministradas por el fiscal del Tribunal Supremo en sus informes anuales, en los que se presentan las drogas decomisadas y los procesos sustanciados por su consumo y tráfico. La edad de los consumidores en porcentaje del total son:

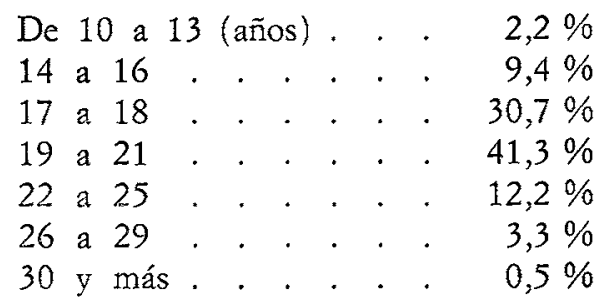

en 1954, y a $41 \%$ en 1956. Véase F. A. Fernández, Aspectos socioculturales del alcobolismo en España (Córdoba: Seminario sobre Alcoholismo, mimeo).

25. J. Santo Domingo et al., op. cit., p. 89. E. Roldán y J. Santo Domingo, El alcobolismo en la mujer española (Madrid: PANAP, 1968).

26. INE, Anuario estadístico de España, 1973. 
«Papers»: Revista de Sociología

\section{TABLA 2}

Drogas decomisadas y procesos incoados por su consumo y tráfico

\begin{tabular}{lrrrrrr}
\hline & 1968 & 1969 & 1970 & 1971 & 1972 & 1973 \\
\hline Drogas & & & & & & \\
$\quad$ Cannabis (Kg) & $\ldots$ & $\ldots$ & 586 & 717 & 895 & 3625 \\
$\quad$ LSD (dosis) & $\ldots$ & $\ldots$ & $\ldots$ & 1171 & 1525 & $\ldots$ \\
Procesos & 308 & 601 & 1249 & 1297 & 1350 & $\ldots$ \\
\hline
\end{tabular}

Fuente: Informes Anuales del Fiscal del Tribunal Supremo. Nota: (...) Sin datos.

Tampoco es firme la base estadística sobre la que operan las organizaciones internacionales; basándose en los datos sobre consumo de sustancias capaces de generar dependencia, las estimaciones de las cifras de dependientes basadas en informaciones policíacas u otras y los informes de los expertos podemos tener una idea de la tendencia del problema y su localización, geográfica o social. El consumo de sustancias capaces de generar dependencias ha aumentado en todo el mundo. Como sustentación de este aserto ofrecemos la evolución del consumo de algunas sustancias determinadas en los países más consumidores de las mismas. Cuando aparece en el mercado una sustancia capaz de farmacodependencia, aumenta su consumo extraordinariamente sin razones terapéuticas que lo justifiquen (véase a este respecto la Tabla 3). Sin que podamos basarnos en datos recientes, podemos afirmar que el problema continúa en pie con los matices propios de las zonas geográficas y culturales en los que se da.

Las autoridades sanitarias abordan el problema de la dependencia con estimaciones basadas en las intervenciones policíacas y en el ingreso de enfermos en los hospitales. Siguiendo estos criterios se han realizado las estimaciones que aparecen en la Tabla 4.

La estimación de Bewly para el Reino Unido, en 1966, ${ }^{27}$ sitúa en 2.400 los usuarios de morfina-heroína, en 24.000 los usuarios de cannabis, en 160.000 los que utilizan anfetaminas con fines extramédicos y en 600.000 los que emplean barbitúricos de los cuales 500.000 los consumen en régimen de dependencia. Finalmente el autor citado calcula que

27. T. Bewley, «Alcoholism», Bulletin of Narcotics, 18 (1966): 241-247. 
TABLA 3

Consumo de diversas drogas

PETIDINA (Kilogramos por $100.000 \mathrm{~h}$.) (a)

USA

\begin{tabular}{|c|c|c|c|}
\hline $1953-57$ & $1963-67$ & 1958.62 & 1967 \\
\hline 48 & 50 & 52,5 & 54,7 \\
\hline 48 & 50,2 & 54,1 & 51,6 \\
\hline 23,3 & 30 & 36 & 39,4 \\
\hline 46 & 43,5 & 43 & 32,6 \\
\hline 42,2 & 33 & 25 & 30 \\
\hline 39 & 22 & 19,1 & 20,08 \\
\hline 13,5 & 16,6 & 16,7 & 16,9 \\
\hline 22,6 & 20,9 & 19,2 & 16,8 \\
\hline
\end{tabular}

DEXTROMORAMIDA (Kilogramos por $100.000 \mathrm{~h}$.) (b)

\begin{tabular}{llll} 
& 1958 & & 1967 \\
\cline { 2 - 2 } Holanda & 1,07 & & 1,67 \\
Francia & 1,01 & & 1,35 \\
Bélgica & 1,44 & & 1,15 \\
Dinamarca & 0,44 & & 1,02 \\
Suiza & 0,38 & & 0,49
\end{tabular}

FENMETRACINA (millones de comprimidos) (c)

\begin{tabular}{lllr} 
& 1964 & & 1958 \\
\cline { 2 - 2 } Suecia & 8,4 & & 20 \\
Checoslovaquia & 0,18 & & 19 \\
Polonia & 0,001 & & 1
\end{tabular}

Fuentes: Organización de Control de Estupefacientes (Ginebra, 1970); Actas del Congreso Mundial de Parmacodependencia (Praga, 1966).

Notas: (a) 1953-1957 consumo medio

1958-1962 consumo medio

1963-1967 consumo medio

(b) Palfium.

(c) Preludín

300.000 habitantes del Reino Unido son alcohólicos (crónicos o no crónicos). 
«Papers»: Revista de Sociología

\section{TABLA 4}

Número y tasas de adictos conocidos

(por millón de habitantes)

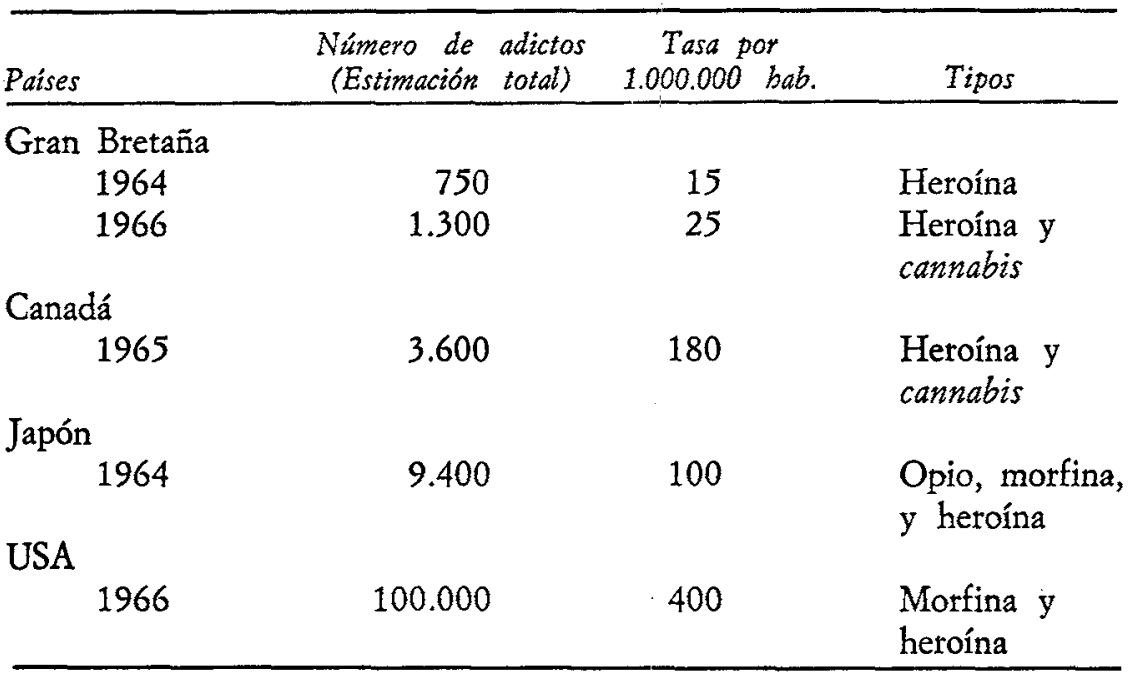

Fuente: United Nations, Commision on Narcotics Drugs (Nueva York; ONU, 1970).

El Comité de Estupefacientes sueco dirigido por Goldberg, basándose en datos procedentes de hospitales y centros de desintoxicación, ha calculado que el $59 \%$ de los adictos abusan de somníferos y tranquilizantes, $18 \%$ de estimulantes, $5 \%$ de productos volátiles inhalados, $4 \%$ de estupefacientes y $1 \%$ de cannabis. Estos datos son concordantes con los procedentes de los ficheros policíacos tanto en su distribución porcentual como en la edad de los enfermos (62\% tenían menos de 30 años, y el $21 \%$ menos de 20 , siendo la edad media de 37,8 años). ${ }^{28}$ «En una prospectiva nacional realizada en enero de 1953 la oficina de Narcóticos registró 28.514 adictos de los cuales $77,8 \%$ usaban heroína; 9,8 morfina; 1,5 opio; 6,3 drogas sintéticas; 4,5 marihuana, y 0,1 cocaína. En un estudio realizado en Chicago en 1951 basado en los registros oficiales de toxicómanos se encontró que el $90 \%$ usaban heroína y el resto principalmente marihuana; el $83 \%$ de los dependientes eran varones y el $50 \%$ menores de 25 años. Analizado un grupo de enfermos bajo tratamiento médico en sanatorios o clínicas privadas se encontró

28. G. Varenne, El abuso de las drogas (Madrid: Guadarrama, 1973). Edición original en francés, 1972. 
que $83 \%$ eran varones de los cuales el $75 \%$ había llegado a niveles universitarios en sus estudios. En Chicago, antes de la Segunda Guerra Mundial, de 2.349 casos sólo el $1 \%$ eran varones menores de 20 años. Alrededor de las dos terceras partes de raza blanca y en la cuarta parte hijos de emigrantes. ${ }^{29}$

Australia, Bélgica, Chile, Dinamarca, Estados Unidos, Francia, Sudáfrica (población blanca), Suiza y la URSS tienen problemas de alcoholismo. El abuso de narcóticos es importante en USA, Hong-Kong, India, Irán, Japón y Tailandia; África, Brasil, India y Pakistán consumen profusamente cáñamo; las hojas de coca constituyen el problema de Bolivia y Perú. El abuso de estimulantes se centra en USA, Japón, Reino Unido, Suecia y Suiza. En algunos países europeos (Alemania, Austria, Suecia) es considerable el abuso de analgésicos menores. En el Extremo Oriente es típico el fumadero de opio.

La variable tiempo también se refiere al aumento en cantidad, calidad y extensión. La oficina de narcóticos norteamericana controlaba en 1953, 30.000 enfermos; en 1962, 79.000; las cifras estimadas para 1966 sitúan en más de 100.000 los posibles dependientes de otras drogas, no del alcohol. Por lo que se refiere al alcohol se estima que el $70 \%$ de los norteamericanos bebe algún tipo de alcohol frente al $4 \%$ de los años 1930, existiendo 4.700 .000 alcohólicos en $19600^{30}$

El consumo de sustancias capaces de engendrar dependencia se ha ampliado, manteniéndose el consumo de alcohol y estupefacientes «clásicos» y aumentándose las anfetaminas, los barbitúricos y los alucinógenos. En 1954, de acuerdo con una encuesta realizada por el Ministerio de Asistencia Social, en Japón de 123.142 personas 9.107 (es decir, un $7,2 \%)$ tomaba anfetaminas y $2.241(1,8 \%)$ abusaban de ellas. El fenómeno empezó en 1948, y en 1955 se calculaba en millón y medio la cifra de usuarios; ;1 a partir de entonces, por las medidas tomadas se controló el problema espectacularmente.

29. S. K. Weinberg, Social Problems in Our Time (New Jersey: Prentice Hall, 1960).

30. M. Keller, «The Definition of Alcoholism and Estimation of Its Prevalence» pp. $24 \mathrm{y}$ ss. en D. J. Pittmann y Ch. R. Snyder (eds.), Society, Culture, and Drinking (Nueva York: Wiley, 1962).

31. OMS, Serie de Informes Técnicos, 102 (Ginebra: OMS, 1956). 


\section{Etiología de las dependencias}

Hablar de etiología de las dependencias es una simplificación excesiva; no existe una teoría coherente que agrupe todos los datos. Las teorías fisiopatológicas sitúan en alguna anomalía corporal, o biológica, el punto de partida de la dependencia. Ésta, y específicamente el síndrome de abstinencia, han llevado a pensar en un defecto metabólico que exigía, incluso sintomáticamente, ser llenado por el tóxico. La similitud del cuadro con los trastornos congénitos del metabolismo, que operan en el mismo sentido, sugirió la búsqueda del eslabón entre droga y dependencia. ${ }^{32}$

Dejando a un lado los estudios primitivos que suponían deficiencias tiroideas o suprarrenales para explicar el alcoholismo, son los estudios de Mardones y la escuela de Chile, Willians y Westerfield los que más interés presentan. Mardones en Chile ${ }^{33} \mathrm{y}$ Willians en USA, ${ }^{34}$ a partir de la preferencia que tienen las ratas hipovitámicas por el alcohol frente al agua, establecen que la base del alcoholismo estaría en la incapacidad genética de utilizar los carbohidratos como fuente de energía rápida. El alcohol sería el sustituto de este defecto enzimático. Desarrollando estas ideas, Westerfield ${ }^{35}$ encuentra diferencias significativas en los circuitos bioquímicos de las ratas bebedoras y no bebedoras de nacimiento, en lo que se refiere a la posibilidad de metabolizar la glucosa, utilizar el acetato y transformar el alcohol en grasas, más rápido en las bebedoras. La extensión de estos estudios a enfermos alcohólicos ha permitido a Warburg ${ }^{36}$ encontrar la existencia de un enzima dehidrogenasa alcohólico atípico que reemplaza al típico en el $5 \%$ al $20 \%$ de los casos. Este enzima tiene una actividad catálica mayor que la normal. Igualmente Feldstein pudo encontrar en el hígado humano tres isoenzimas de la

32. Así como la falta de insulina provoca la necesidad de comer y beber líquidos en exceso, la falta de un factor desconocido provocaría la necesidad de beber alcohol, y padecer su intoxicación aguda y crónica.

33. R. J. Mardones, «On the Relations Between Deficiency of B Vitamin and Alcohol Intakes in Rats», Quarterly Journal of Studies on Alcobol, 12 (1951): 563-571.

34. R. Williams, Nutrition and Alcobolism (Norman: Oklahoma University Press, 1951).

35. W. Westerfield, «Effects of Caloric Restrictions and Deficiency on Voluntary Consumption of Alcohol by Rats», Quarterly Journal of Studies on Alcobol, 14 (1953): 378-390.

36. J. P. Warburg, «Methabolism of Ethanol», Veintiocho Congreso Internacional sobre Alcobol y Alcobolismo (Washington: mimeo, 1968). 
alcohólico dehidrogenasa que no se reparte por igual por toda la población. ${ }^{37}$

Todos estos estudios de innegable valor chocan con tres dificultades básicas: los alcohólicos crónicos tienen una alteración hepática que enmascara todos los hallazgos a los que quiera darse valor anterógeno. Los experimentos en ratas tienen una limitación enorme al extenderse al hombre. Para que estos estudios tengan valor holístico deberán acompañarse de hallazgos parecidos para explicar la dependencia de otros tóxicos tan poderosos o más que el alcohol.

Las teorías psicopatológicas mantienen que el dependiente de las drogas padece un trastorno de la personalidad o un desequilibrio afectivo. Ciertamente, basándose en los rasgos caracterológicos de los enfermos, Lisanzky ${ }^{38}$ afirma que los individuos predispuestos tendrían los rasgos siguientes: fuerte necesidad de permanecer dependientes de sus progenitores, inadecuados mecanismos de defensa, intenso conflicto entre el deseo de ser infante y necesidad de ser adulto, baja tolerancia a las frustraciones y ambivalencia en sus amores-odios. Los psicoanalistas destacan la personalidad morbosa, oral y narcisista de los enfermos dependientes. Ahora bien, la personalidad prealcohólica, o predependiente, no existe como tal sino sencillamente como personalidad inmadura, pudiendo expresar su inmadurez como dependencia o de cualquier otro modo. Todos los estudios sobre personalidad en dependientes se han realizado a posteriori $\mathrm{y}$, de «igual modo que la demencia senil acaba homogeneizando a todos los seniles, el deterioro del alcoholismo redondea las aristas $\mathrm{y}$ vértices que hasta entonces han compuesto los matices de su propia personalidad». ${ }^{39}$

Las personas que utilizan drogas pueden clasificarse en cuatro grupos: (a) Individuos emocionalmente bien ajustados, que utilizan las drogas para el tratamiento médico del dolor, o por placer. (b) Individuos que utilizan las drogas para compensar su personalidad neurótica. (c) Individuos que utilizan las drogas buscando deliberadamente sus efectos patológicos. Psiquiátricamente, pueden ser clasificados como psicópatas. (d) Individuos relativamente normales, que utilizan las drogas como medio

37. A. Feldstein et al., «Is Alcohol Dehidrogenase an Etiological Factor in Alcoholism?», Veintiocho Congreso Internacional sobre Alcobol y Alcobolismo (Washington: mimeo, 1968).

38. E. S. Lisansky «The Etiology of Alcoholism: The Role of Psychological Predisposition», Quarterly Journal of Studies on Alcobol, 21 (1960): 314-322.

39. C. Castilla del Pino, «Problemas psicosociológicos del alcoholismo», Salud Mental, 5 (1969): 9-17. 
para seguir en un subgrupo marginal. Así los adolescentes, delincuentes juveniles, etc.

En comparación con la población media, entre los dependientes encontraremos más personalidades inmaduras, pero la semejanza en los rasgos de la personalidad de los tóxico-dependientes no concuerda con el hecho de que personalidades manifiestamente promedias puedan hacerse dependientes en ciertas situaciones de tensión especial o ciertas situaciones ambientales, como cuando los taberneros se hacen alcohólicos, o los médicos morfino-dependientes.

\section{El significado de las drogas en la sociedad}

En todas las sociedades encontramos alguna sustancia para evitar el dolor o la fatiga o para provocar la euforia. El alcohol es sin duda la más extendida de ellas. En las sociedades donde no existe el alcohol, el opio, la cocaína o el peyote ejercen un papel similar. Podríamos decir que cada cultura elige su droga y la regula limitando su utilización a ciertas situaciones sociales conocidas y predeterminadas. ${ }^{40}$

La elección de un tipo u otro de droga es también un hecho cultural vinculado a su disponibilidad, a la capacidad técnica de obtener el producto activo y a la transmisión de las técnicas de su obtención. Por ello pueden delimitarse zonas de mayor influencia de un tóxico coincidiendo con los lugares geográficos en que la producción permite el fácil acceso al producto. Así sucede por ejemplo con el vino, la cerveza, el opio y los alucinógenos.

Tanto los estudios empíricos como los análisis teóricos coinciden en señalar que la sociedad facilita la disponibilidad de las drogas a la vez que delimita, regula y dictamina su utilización. El consumo de drogas ( $y$ en concreto de una determinada) se aprende, e incluso se impulsa a su consumo, en las situaciones previstas permitiendo que se conozcan los efectos farmacológicos en un proceso de iniciación.

Las drogas pueden utilizarse para fomentar un sentimiento de tranquilidad, libertad o relajación. En algunas culturas esto puede significar tomar una copa antes de cenar y en otras una pipa de kif al terminar la cena. Estrechamente ligado con esto está el uso de la droga como parte de un rito social; por ejemplo, tomar una copa o fumar una pipa juntos como prueba de amistad; pertenencia a un grupo restringido o

40. M. M. Glatt, «Problemas comunes al alcoholismo y a la dependencia de las drogas», Crónica de la OMS, 21 (1967): 312-323. 
de adhesión a los conceptos e ideales de un grupo más amplio. Los usos mencionados se aceptan respecto al alcohol y la cannabis en diferentes culturas, aun cuando estas sustancias puedan estar oficialmente prohibidas. El uso cultural de drogas es más amplio que el meramente religioso en cuyas ceremonias la utilización de estos productos es obvia.

Como señala Horton para el alcohol, ${ }^{48}$ la función primaria de las drogas en la sociedad es reducir la ansiedad, unas veces de modo manifiesto, como en el caso de sociedades primitivas, otras de modo latente como en las que utilizan este medio para manifestar solidaridad.

Las drogas cumplirían así el papel de ansiolítico social en un doble sentido: de solucionador neurótico de las tensiones y mecanismo tolerado en el medio en el que actúa. Quiere esto decir que el consumo de tóxico es querido por la sociedad, que acepta de este modo la evasión de sus problemas sancionando como mecanismo alternativo de relajación el uso de una droga, la que coincide con la máxima accesibilidad.

$\mathrm{La}$ apelación mayoritaria a un tóxico para liberar tensiones viene también determinada por la falta de otros mecanismos alternativos de idéntica finalidad. Como señala Bales, ${ }^{42}$ la cultura influencia el alcoholismo de tres modos: creando la tensión, tolerando el consumo para aliviarla y no suministrando alternativas válidas de solución. Es claro que si el mecanismo ofrecido no es alcohol sino otro tóxico, la dependencia creada será otra.

\section{La bipótesis de la anomia en la dependencia de las drogas}

Merton al afirmar que «las estructuras sociales ejercen una presión definida sobre ciertas personas en la sociedad induciéndolas a una conducta de rebeldía antes que conformidad» ${ }^{43}$ extendió la potencialidad explicativa de la anomia desde el suicidio a otros tipos de conducta desviadas, y conectó la anomia con la conducta divergente a través de un proceso social caracterizado.

En la estructura social se distinguen, según Merton, las metas y los medios institucionalizados para alcanzarlas o, to que es lo mismo, la estructura cultural —objetivos, propósitos e intereses culturalmente definidos, sustentados como legítimos por todos los miembros de la socie-

41. D. Horton, «The Function of Alcohol in Primitive Society: A Cross-Cu! tural Study», Quarterly Joumal of Studies on Alcohol, 4 (1943): 191-230.

42. R. F. Bales, "Cultural Differences in Rates of Alcoholism», Quarterly Journal of Studies on Alcobol, 6 (1946): 480-499.

43. R. K. Merton, Teoría y estructuras sociales (México: FCE, 1964). 
dad- y la estructura social -reglas arraigadas en las costumbres e instituciones relativas a los procedimientos permisibles para alcanzar dichos objetivos.

Estos dos elementos estructurales no están en relación constante o directa. Puede suceder que el éxito no esté al alcance de todos los miembros de la sociedad a través de los medios legítimos y es justamente este desequilibrio el que da lugar a la anomia. Así, dice Merton, «la cultura puede ser tal, que induzca a los individuos a centrar sus convicciones emocionales sobre el complejo de fines culturalmente proclamados, con mucho menos apoyo emocional para los métodos prescritos para alcanzar estos fines». ${ }^{44}$ En estas condiciones es el procedimiento más eficaz el que se elije atenuándose las normas.

Paradójicamente, las mismas estructuras social y cultural que fundamentalmente conforman y organizan las conductas generan también tendencias hacia determinados tipos de desviación y son potencialmente fuentes de desorganización social. En este ambiente cultural los individuos responden de forma preestablecida de tal manera que su conducta se encuentra abocada a adaptarse a esta situación social anómala. Merton elabora una tipología de adaptación individual que incluye cinco tipos o categorías (conformidad, innovación, ritualismo, retraimiento y rebelión) - de acuerdo con la aceptación o rechazo de las metas de la sociedad anómica. ${ }^{45}$ En este marco conceptual, más aún si entendemos anomia en un sentido amplio y no la limitamos a las barreras estructurales que se oponen a la realización de los objetivos de éxito (fuente principal de la anomia en nuestra sociedad, pero en modo alguno única), la depen-

44. Ibid., pp. 143 y ss.

45. Ibid., pp. 149 y ss.

46. Es el error en el que caen a mi juicio A. Lindesmith y S. A. Gagnon en su artículo sobre «Toxicomanías y anomia» pp. 151-179 en M. B. Clinard (ed.), Anomia y conducta desviada (Buenos Aires; Paidos, 1967). Al limitar la fuente de anomia al bloqueo, incluso en doble fracaso, en el camino al éxito han de concluir que la teoría no es aplicable a la mayoría de los aspectos de la conducta del uso de drogas. Es curioso que, sin embargo, Ch. R. Snyder en la misma recopilación encuentre su aplicabilidad al alcoholismo, pero este autor no limita a una la fuente de anomia. No se argumente que el concepto mertoniano es estricto, porque también Merton ha escrito "cuando se establece un alto grado de anomia, las reglas han perdido su sabor y su fuerza. [...] Pues ya no hay un sentido. [...] Un cierto grado de anomia se ha producido en las masas de neoyorkinos [...] cuando ya no pueden confiar en no ser atacados por sus compañeros de viaje. [...] Cuando vacilan en frecuentar parques por temor a ser asaltados. [...] Cuando las gentes temen ser engañadas, embaucadas. [...] Pues si no se comparten las normas uno no puede saber qué ha de esperarse del otro y ésta es una condición social admirablemente apropiada para producir relaciones inseguras con otros» (pp. 212 y ss.). 
dencia de las drogas es un modo de retraimiento al que puede llegarse bruscamente o mejor a través de adaptaciones intermedias de otros tipos, pues el retraimiento es un final al que pudo llegarse directamente 0 tras el ritualismo o la rebelión. .7 $^{2}$

Sea como sea, los factores biológicos, psicológicos y sociológicos operan dialécticamente a nivel de la conciencia individual de la persona, para la cual la búsqueda de la droga, con la dependencia cada vez más fuertemente establecida, es el final común de múltiples situaciones tensionales como vía alternativa de respuesta a las mismas. La ingestión de la droga es la solución más económica en la situación concreta en la que el sujeto se encuentra, si bien se trata de una solución subjetiva y falsa, y en consecuencia contraproducente. Mediante la desconcienciación que la droga provoca, el sujeto elude la posibilidad de una conducta que repare la situación conflictual originaria. Al conflicto por el que se toma la droga se añade ahora el conflicto por tomarla. La dependencia de la droga no estriba tanto en las modificaciones que hayan tenido lugar por su ingestión, cuando por el hecho de que ante la progresiva complicación del conflicto originario, las posibilidades de solución se alejen más y más $\mathrm{y}$, por consiguiente, la necesidad de evasión es cada vez más imperiosa. A la mayor necesidad de huir de la realidad se une, ahora como antes, la fácil disponibilidad de la droga usual. ${ }^{\text {ts }}$

\section{La lucba contra las dependencias}

Desde el punto de vista general, las acciones que pueden emprenderse contra este problema se desarrollan en tres niveles de profundidad: evitar su presentación," tratar a los afectados y consolidar su curación; o lo que es lo mismo, prevención primaria, secundaria y terciaria.

47. La tipología de Merton es poco aplicable a la adaptación individual, pues ésta puede variar. El propio Merton, sin embargo, afirma: «Hay un cargo de innovador, ritualista, retraído y rebelde en la mayoría de nosotros. Que esos rasgos lleguen a manifestarse de modo abierto y luego se repitan hasta hacerse crónicos depende tanto de las respuestas de quienes nos rodean como de nuestro propio carácter y personalidad.» Ibid., p. 206.

48. Esta es la idea central de C. Castilla del Pino en «Problemas psicosociológicos del alcoholismo». Los conceptos están aplicados al alcohol, pero sin forzarlos pueden, y deben, extenderse a todas las drogas.

49. Más realista sería el reducir la incidencia y la gravedad de los problemas asociados con la dependencia. 


\section{Prevención primaria}

La disminución de la incidencia llegará por un triple camino: reducir las oportunidades de adquisición de droga, eliminar las condiciones por las que el individuo expuesto las utiliza, convencer a los expuestos a que no las consuman.

\section{Acciones sobre las drogas}

La única acción preventiva frente a la droga es mantenerla bajo supervisión administrativa en su producción, distribución y venta. En teoría cabe también la restricción absoluta, como en el caso de la Ley Seca, pero este tipo de maximalismos, casi siempre promovidos desde posturas moralistas, olvidan que las drogas son una salida alternativa a las tensiones sociales y su cierre implicaría el desbordamiento de otras. ${ }^{50}$ Por otro lado no será nunca posible restringir de forma total la imaginación del ser humano para usar sustancias psicótropas y, en definitiva, las drogas-medicamento nunca pođrán restringirse.

La fiscalización de un fármaco puede ser necesaria siempre que se cumpla al menos una de las dos condiciones siguientes: (1) Cuando se sepa que el empleo abusivo de la sustancia no es sólo esporádico o localizado, que sus efectos no se limitan al usuario, que su difusión implica una comunicación entre el drogado y los consumidores potenciales y que empieza a establecerse un tráfico ilícito de la droga en cuestión. (2) Cuando se proyecte utilizar el fármaco en medicina y los datos experimentales muestren un riesgo notable de engendrar una dependencia física o psíquica y el fármaco haya sido o pueda ser comercializado. ${ }^{51}$

Para conseguir la fiscalización funciona en todos los países una unidad de control, generalmente en el departamento competente en salud pública que fiscaliza los productos toxicomanígenos. La coordinación de estas acciones a nivel internacional se realiza por la Comisión de Estupefacientes del Consejo Económico y Social de las Naciones Unidas. En este sentido, se desarrollan experiencias controladas para intentar medir el riesgo de dependencia tanto en animales como en personas

50. Los intentos enérgicos para imponer la prohibición frente a una demanda son irreales y poco útiles como se comprobó en USA y Noruega con el alcohol y en Irán y Tailandia con el opio.

51. OMS, Serie de Informes Técnicos, 407 (Ginebra: OMS, 1964). 
voluntarias. Los trabajos realizados en el Addiction Research Center en Lexington (USA) sirven de pauta. Cuatro tipos de experiencias son básicos: evaluación de los efectos de dosis únicas administradas por una o más vías; pruebas de sustitución en pacientes con dependencia; estudios de dependencia directa, y pruebas para ver la preferencia de las diferentes vías.

Todo fabricante de un medicamento analgésico debe informarse si su producto es susceptible de engendrar dependencia con objeto de someterlo a fiscalización cuya ejecución práctica puede revestir dos modalidades: venta con receta médica (menos rigurosa) y venta con recetacarnet de estupefacientes (máximo rigor).

Cuando una droga no es medicamento, una serie de medidas legislativas de mayor o menor alcance restringen las facilidades de su obtención y sancionan a los que trafican con ellas. ${ }^{52} \mathrm{Al}$ respecto y desde el punto de vista médico conviene hacer las siguientes precisiones: es una incongruencia «perseguir» consumo de una droga como la cannabis ${ }^{33}$ y dejar en venta libre el alcohol; las sanciones no impiden que los individuos dependientes reanuden su comportamiento; es ilógico considerar a los dependientes como delincuentes y no como enfermos. ${ }^{54} \mathrm{La}$ persecución del traficante es lógica, pero cuando el dependiente es a la vez traficante debe ser tratado como enfermo. Los precios elevados no disuaden a los dependientes, sencillamente se consigue el dinero por medios ilícitos o se toma un producto degradado. Las sanciones penales graves por la posesión de pequeñas cantidades de droga son improce-

52. Dentro de estas medidas se encuentran: aumentos de impuestos que gra. van el consumo, reducción de horarios de venta, venta en expendedurías, control aduanero, persecución policíaca y penas de cárcel. Siempre hay una droga aceptada que «desaloja» a las otras; entre nosotros el alcohol.

53. Que debe, a mi juicio, ser fiscalizada pues, «como han señalado los precedentes Comités de Expertos de la OMS, que se ocupan de la farmacodependencia, no existe en la actualidad ninguna necesidad de utilizar la cannabis como medicamento. Sin embargo, el consumo no médico de esta sustancia persiste e incluso ha aumentado en algunos países. En ciertos países se observan opiniones muy divergentes sobre la capacidad del cannabis para provocar dependencia, así como de los efectos agudos y crónicos de la droga sobre el usuario y la colectividad y sobre el tipo y la naturaleza de las medidas de fiscalización que se han de aplicar. EI Comité afirma enérgicamente las opiniones expresadas en anteriores informes, es decir, que la cannabis «es una droga capaz de engendrar y de plantear problemas de salud pública y sociales por lo que deben mantenerse las medidas de fiscalización", OMS, Serie de Informes Técnicos, 516 (Ginebra: OMS, 1973).

54. Tanto es así que en el Reino Unido y los USA se ha comprobado la utilidad de combatir la dependencia de una droga fuerte - heroina - con otra menor - metadona - suministrada oficialmente. 
dentes, debe hacerse todo lo posible por quitar el carácter delictivo al consumo. 5 .

El control del producto, aunque valioso, no puede resolver la totalidad del problema, por severa que sea, ninguna restricción de la producción, la distribución, y la administración de drogas puede resolver por completo el problema de la prevención. En términos epidemiológicos las medidas preventivas no deben limitarse al agente, sino que deben extenderse al huésped y al medio dirigiéndose así a las causas subyacentes de la adición.

Las condiciones socio-económicas adversas y otros factores ambientales se asocian a menudo con el uso de drogas. Miseria, desamparo, discriminación o tensiones profundas empujan a muchos al consumo de drogas supuesta su disponibilidad. Hay que comenzar una acción destinada a modificar los sistemas políticos y estructuras sociales que mantienen los estímulos nociceptivos. Esta acción reformadora nos sitúa en una esfera en la que al menos una es competencia médica: ofrecer consejo a los legisladores, a los líderes de la comunidad y colaborar con otros ciudadanos para cambiar democráticamente las normas e instituciones.

Frente al medio social, además de su reestructuración profunda, cabe la modificación de las actitudes hacia las drogas toleradas, o al menos su no favorecimiento por medio de la supresión de su propaganda, restricción de la fascinación con que se rodea la participación en actividades relacionadas con las drogas y reprobación pública de su consumo. Aunque no sea probable que la prohibición (incluso total) de los anuncios de drogas destinadas al público en general baste para resolver los problemas asociados al uso del alcohol y de otras drogas causantes de dependencia, incumbe a quienes se benefician con las ventas de esas sustancias ( $\sin$ excluir a los gobiernos), la obligación de explicar por qué la sociedad debe seguir tolerando lo que es probablemente un factor de agravación. En este orden de ideas es una contradicción

55. Compárense estas ideas con el siguiente párrafo: «En Madrid, la Brigada de Estupefacientes en noviembre último captura una banda de jóvenes. [...] El núcleo o centro del grupo es un individuo de 22 años. [...] En un tiempo simpatizó con el fascismo. Luego se hace comunista pro-Moscú [sic]. Más tarde se siente anarquista y destructor de la sociedad [sic] [...] Al ser detenido se le ocupan varias partidas de camabis. [...] Las reuniones del grupo son bacanales. La inversión sexual es admitida como algo natural [sic] [...] De 49 jóvenes interrogados, 30 admiten haber consumido drogas adquiridas al sujeto en cuestión que fue quien les llevó al vicio [sic].» J. M. Mato, «Propagación epidémica en España del uso indebido de drogas», p. 239, en Alcobolismo y otras toxicomanias (Madrid: PANAP, 1970), pp. 234-245. 
la propaganda de bebidas alcohólicas en los medios de información dependientes de la administración pública.

\section{Actividades dirigidas bacia los expuestos}

Desde el momento en que cada sociedad tolera una droga, toda la población debe considerarse como expuesta. Ahora bien, es preciso distinguir entre los que están expuestos a un riesgo elevado y aquellos en los que no existe ese nivel. Sobre ambos caben acciones de información y educación sanitarias que tengan en cuenta que el material destinado a informar puede incitar a la experiencia con drogas a personas poco estables, por lo que, si bien la ignorancia es un mal, no obstante no parece conveniente emprender programas intensivos sobre una droga donde no exista un problema real o potencial.

Habida cuenta de que los medios de información colectiva procuran muy legítimamente satisfacer la curiosidad acerca de los fenómenos sociales nuevos, es importante que se utilicen datos fidedignos sobre cuestiones como la farmacodependencia; de lo contrario, se corre el riesgo de difundir material sensacionalista. La presentación de los hechos es tan importante como su precisión. «Es evidente la necesidad de que las autoridades sanitarias y las personas encargadas de los medios de información colectiva trabajen en estrecha relación para proporcionar datos más precisos acerca de las drogas y la farmacodependencia, decidir las actitudes que deben fomentarse para servir a la sanidad sin excitar al público, alejar el peligro de que las emisiones de radio y televisión y los artículos de la prensa tengan efectos nocivos y evaluar la eficación de los esfuerzos desplegados. $\rangle^{56}$

\section{Prevención secundaria}

La historia natural de la dependencia de las drogas tiene dos momentos críticos: su existencia presintomática y sus comienzos como tal. En estos momentos la enfermedad es más vulnerable y los esfuerzos desplegados para cortarla poseen las máximas posibilidades de éxito. El tratamiento intensivo y precoz es el instrumento más apropiado de la prevención secundaria. Como tal se edifica en tres principios: desinto-

56. Oficina Regional de la OMS para Europa, Farmacodependencia: Prevención y lucba (Copenague: OMS, 1971). 
xicación por la supresión brusca y total, ${ }^{57}$ refuerzo de la personalidad por psicoterapia y sostenimiento posterior en un plan a largo plazo.

El tratamiento precoz implica operacionalmente el diagnóstico precoz que puede conseguirse a través de: búsqueda de sospechosos para ofrecerles ayuda y tratamiento, vigilancia de los síntomas claves como mallas de síntomas de alcohólicos, huellas de pinchazos de agujas, hepatitis postinyecciones en poblaciones expuestas y difusión de síntomas precoces entre personal sanitario, trabajadores sociales o autoridades judiciales. Todo ello, sin embargo, es inútil sin la paralela consecución de una actitud más comprensiva, que fomente la consideración de las dependencias como una enfermedad y faciliten la oportuna consulta y tratamiento.

\section{Prevención terciaria}

La farmacodependencia, especialmente la de los tipos alcohol-barbitúricos y opiáceos, tiende a ser un trastorno recidivante y crónico. El pronóstico (a largo plazo) de esta enfermedad es en general bastante negativo. Por ello la mejor parte del trabajo frente a ella se ha planteado en los niveles preventivos anteriores. La mejora en la eficacia y disponibilidad de aparatos de tratamiento y sostenimiento de la abstinencia permiten un mejor pronóstico, y a ello colaboran intensamente todas las acciones que buscan consolidar al ex enfermo en la abstinencia.

La mayor dificultad con la que nos encontramos surge del medio social que provocó la enfermedad y al que el enfermo vuelve. La modificación del medio social es, a la vez que primaria, prevención terciaria. Por desgracia su alcance es muy limitado. Por ello el camino más viable discurre por el sostenimiento del ex enfermo y su evolución positiva, en lo que se refiere a la satisfacción personal, la imagen de sí mismo y a las realizaciones individuales, pues lo que se busca no es solamente prevenir las recaídas sino el mejoramiento general de sus relaciones interpersonales. ${ }^{s 9}$

A conseguir esta finalidad contribuyen los servicios a medio camino entre el hospital y la comunidad ${ }^{59}$ que tienen más de asistencia social

57. Entiendo que no es de interés señalar aquí la forma concreta de la supresión; sólo indicar que los psiquiatras aceptan un ritmo más lento de supresión en opiáceos y barbitúricos y que en el caso de la heroína hay un paso intermedio de sustitución por metadona.

58. OMS, Serie de Informes Técnicos, 551 (Ginebra: OMS, 1974).

59. Hogares postcura, clubs de ex enfermos, tefugios, alojamientos y servicios de acogida. No en todos los casos hay necesidad de hospitalización. 
que de enfermería, tratando de prestar apoyo a los pacientes dados de alta, de mantener el lazo terapéutico con el centro médico y de facilitar sus primeros pasos en la familia. En esta tarea se han mostrado también eficaces las asociaciones de ex enfermos que han ayudado a gran número de dependientes."

En resumen, la dependencia de las drogas es una enfermedad recurrente, la recaída no debe considerarse como un fracaso en el tratamiento sino como un reto, y entablar nuevamente la lucha. Al menos así lo vemos los especialistas en Salud Pública.

Francisco Javier Yuste Grijalba

Jefe Provincial de Sanidad

Peregrina, 80

Pontevedra.

60. La más conocida Alcobolicos Anónimos. Éstos deberían funcionar bajo supervisión médica, en todo caso, pues la recaída del líder es la recaída de todo el grupo. 